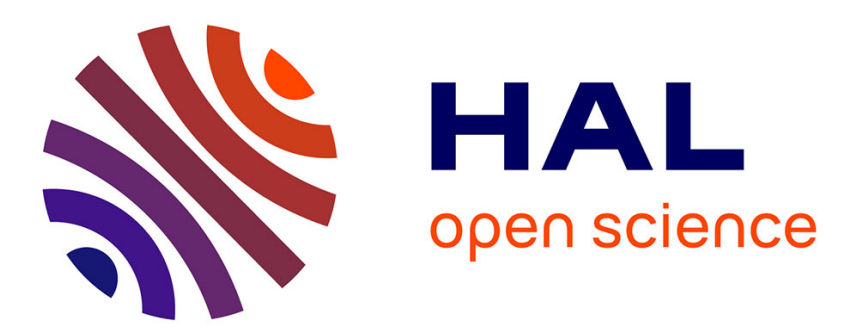

\title{
RhoB loss induces Rac1-dependent mesenchymal cell invasion in lung cells through PP2A inhibition
}

E Bousquet, O Calvayrac, J Mazières, I Lajoie-Mazenc, N Boubekeur, G

Favre, A Pradines

\section{- To cite this version:}

E Bousquet, O Calvayrac, J Mazières, I Lajoie-Mazenc, N Boubekeur, et al.. RhoB loss induces Rac1dependent mesenchymal cell invasion in lung cells through PP2A inhibition. Oncogene, 2016, 35 (14), pp.1760-1769. 10.1038/onc.2015.240 . hal-03026032

\section{HAL Id: hal-03026032 https://hal.science/hal-03026032}

Submitted on 26 Nov 2020

HAL is a multi-disciplinary open access archive for the deposit and dissemination of scientific research documents, whether they are published or not. The documents may come from teaching and research institutions in France or abroad, or from public or private research centers.
L'archive ouverte pluridisciplinaire HAL, est destinée au dépôt et à la diffusion de documents scientifiques de niveau recherche, publiés ou non, émanant des établissements d'enseignement et de recherche français ou étrangers, des laboratoires publics ou privés. 


\title{
ORIGINAL ARTICLE
}

\section{RhoB loss induces Racl-dependent mesenchymal cell invasion in lung cells through PP2A inhibition}

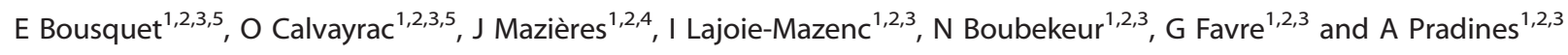

Non-small-cell lung cancer (NSCLC) is the leading cause of cancer-related death worldwide, which is mainly due to its high risk of metastatic dissemination. One critical point of this process is the ability of cancer cells to detach from the primary tumor and migrate through the extracellular matrix; however, the underlying molecular mechanisms are not yet fully understood. In the present study, we identified the small GTPase RhoB as a key regulator of bronchial cell morphology in a three-dimensional (3D) matrix. RhoB loss, which is frequently observed during lung cancer progression, induced an epithelial-mesenchymal transition (EMT) characterized by an increased proportion of invasive elongated cells in 3D. The process was mediated by Slug induction and E-cadherin repression. In addition, downregulation of RhoB induced Akt1 activation, which in turn activated Rac1 through the guanine-exchange factor Trio to control cell shape rearrangement. Further, we provide evidence that RhoB interacted with and positively regulates phosphatase PP2A through the recruitment of its regulatory subunit B55, which was found to be crucial for Akt dephosphorylation. B55 inhibition completely suppressed RhoB-mediated PP2A regulation. Finally, we show that PP2A inactivation, by targeting either its catalytic or its regulatory B55 subunit, completely reversed RhoB-dependent morphological changes and also fully prevented the ability of RhoB to decrease the invasiveness of bronchial cells. Altogether, these results highlight a novel signaling axis and describe new molecular mechanisms that could explain the tumor suppressor role of RhoB in lung cancer. Therefore, we propose that RhoB could be responsible for early metastatic prevention by inhibiting the EMT-derived invasiveness of lung cells through the control of PP2A activity.

Oncogene advance online publication, 6 July 2015; doi:10.1038/onc.2015.240

\section{INTRODUCTION}

The metastatic process causes most of the fatal outcomes in lung cancer patients, and underlines the need for a better understanding of the associated molecular events. During this process, tumor cells interact with their environment and move in threedimensional (3D) structures. The development of metastases is a complex process and involves several cellular and biochemical modifications that trigger the migratory and invasive phenotype. First, cells from the primary tumor undergo epithelial-mesenchymal transition (EMT) induced by several EMT transcription factors (EMT-TFs), such as Snail, Zeb-1 or Slug. ${ }^{1}$ These EMT-TFs notably control the expression of proteins that mediate cell-cell contact, such as E-cadherin, expression of mesenchymal markers such as vimentin and matrix metalloproteinases, and participate in cystoskeleton remodeling. ${ }^{2}$ EMT allows the polarized epithelial cell to be converted into a motile cell that can detach from the primary tumor and invade through the basement membrane into the vasculature. Cells that have undergone EMT can use two modes of migration-mesenchymal migration, in which cells adopt an elongated shape and depend on ECM proteolysis via matrix metalloproteinases' activity, or amoeboid migration, which is characterized by squeezing movements that allow cancer cells to glide through matrix barriers. Cell plasticity, which allows cells to rapidly switch between both states by a so-called amoeboid-mesenchymal transition, depends on microenvironmental changes and matrix stiffness that can occur during tumor progression and therapeutic treatments, ${ }^{3}$ and favors cancer dissemination.

The Rho family members are small GTPases that belong to the Ras family ${ }^{4}$ and are involved in multiple cellular processes including cytoskeleton organization, cell motility, cell adhesion and transcription, and thus are key players of metastatic dissemination. For example, RhoA is well known to stimulate cell migration. ${ }^{5,6}$ RhoA and RhoC expression correlates positively with metastasis in a number of cancer types..$^{5}$ In contrast, Rnd3/RhoE downregulation controls cellular invasion in hepatocellular carcinoma. ${ }^{7}$ RhoGTPases have a dynamic role in EMT, ${ }^{8}$ such as RhoA and RhoC in colon carcinoma ${ }^{9}$ and amoeboid-mesenchymal transition, where Rac1 activation triggers a mesenchymal phenotype, $^{10}$ whereas RhoA regulates the highly contractile amoeboid migration via the ROCK-myosin II signaling axis. ${ }^{11}$

The RhoB protein is distinguishable from its homologs RhoA and RhoC. Indeed, RhoB acts as a tumor suppressor ${ }^{12-14}$ and its expression is frequently downregulated in cancer. ${ }^{15-17}$ We reported that, in lung cancer, RhoB expression was inversely correlated with tumor progression. ${ }^{18}$ Moreover, it has been highlighted, by in vitro and in vivo experiments, that RhoB has a negative role in migration and invasion, ${ }^{19-21}$ which is most notable in human bronchial cells. ${ }^{22}$ We and others have shown that $\mathrm{RhoB}$ regulates the migratory and invasive properties via the regulation of Akt activity in bronchial, ${ }^{22}$ breast $^{23}$ and gastric cancer cells. $^{24}$

${ }^{1}$ Inserm, UMR 1037-CRCT, Toulouse, France; ${ }^{2}$ Université Paul Sabatier, Toulouse, France; ${ }^{3}$ Département de Biologie, Institut Claudius Regaud, Toulouse, France and ${ }^{4}$ Hôpital Larrey Département de Pneumologie, CHU Toulouse, Toulouse, France. Correspondence: Dr A Pradines, Institut Universitaire du Cancer Toulouse - Oncopole, 1 avenue Irène JoliotCurie, 31059 Toulouse, France.

E-mail: pradines.anne@iuct-oncopole.fr

${ }^{5}$ These authors contributed equally to this work.

Received 28 August 2014; revised 12 May 2015; accepted 22 May 2015 
In order to deepen our knowledge on the role of RhoB in metastatic dissemination during lung cancer, we studied the role of this GTPase in lung cell behavior using a 3D approach to decipher the role of RhoB in invasion of bronchial cells and the involved signaling pathway. Data presented herein reveal a novel pathway that controls bronchial cell morphology in a 3D environment and could contribute to a better understanding of the metastatic process in lung cancer.

\section{RESULTS}

RhoB depletion induces EMT in bronchial cell lines and increases cell invasion

To investigate the role of RhoB on cell behavior in 3D culture, we first determined the morphology of several lung cell lines in a 3D matrix. Cells were embedded in droplets composed of a Matrigel/ collagen mixture, which mimicked the pulmonary extracellular matrix. $^{25}$ In this 3D environment, lung cancer cells displayed heterogeneous morphologies classified into three groups: (i) cells that displayed a rounded shape (for example, H520, H460 and H4006); (ii) cells with an elongated fibroblast-like shape with cellular protrusions (for example, Calu-1, H1975 and H1650); and (iii) cells that presented a mixed phenotype (for example, H2935, $\mathrm{H} 23$ and the immortalized bronchial cells BEAS-2B; Supplementary Figure S1A).

The analysis of RhoB expression by western blot and RT-qPCR (quantitative reverse transcription PCR) analysis showed that rounded cells displayed significantly higher levels of RhoB compared with elongated cells (Supplementary Figures S1B and C), suggesting a direct role of $\mathrm{RhoB}$ expression on the $3 \mathrm{D}$ cell morphology.

Thus, we then assessed the effect of modulation of RhoB expression (either by short interfering RNA (siRNA)-mediated RhoB silencing or by adenoviral-mediated RhoB overexpression) on the $3 D$ cell morphology of one selected cell line from each group ( $\mathrm{H} 520$ for rounded cells, Calu- 1 for elongated cells and BEAS-2B as a model for the mixed phenotype). RhoB downregulation by two distinct siRNAs resulted in a significant increase in the percentage of elongated cells in the three cell types, whereas RhoB overexpression induced the opposite effect on immortalized BEAS-2B and Calu-1 cells (Supplementary Figure S2 and Figures 1a and b). RhoB overexpression had no effect on H520 cell morphology as almost all control cells already displayed a rounded morphology (data not shown). Furthermore, the downregulation of $\mathrm{RhoB}$ expression following transformation of the BEAS-2B cells by the oncogenic mutant K-Ras ${ }^{\mathrm{V} 12}$ (Supplementary Figure S2A), as previously shown, ${ }^{22}$ induced a strong increase in the percentage of elongated cells (Figures $1 \mathrm{a}$ and $\mathrm{b}$ ). Consistent with the above observations, all three RhoB-depleted cell lines, as well as the KRas ${ }^{\mathrm{V} 12}$-BEAS-2B cells, showed a marked increase in cell-extension size, whereas adenoviral-mediated RhoB overexpression in BEAS-2B and Calu-1 resulted in a significant reduction in protrusion size (Figures $1 \mathrm{a}$ and $\mathrm{c}$ ).

We previously showed that the loss of RhoB expression promotes invasiveness and metastatic dissemination in vivo of BEAS-2B cells. ${ }^{22}$ Thus, we determined whether RhoB silencing impacts on BEAS $2 B$ cell dynamic movements in the $3 D$ matrix, monitored by time-lapse microscopy. The majority of control cells showed a rounded morphology and slow random movements. In contrast, siRhoB-transfected cells displayed a more elongated shape and moved more quickly with dynamic protrusion extension (Supplementary Figure S3A, Supplementary Movies 1 and 2). The cell shape dynamics was quantified by determining the difference of the Feret's diameter (or maximum caliper length) over time. This parameter was significantly higher in RhoBdepleted cells compared with control cells, and was correlated to the quick formation of protrusions (as shown in Supplementary Figures S3B and C).

Moreover, in a 3D wound-healing assay, we showed that RhoB downregulation induced an elongated morphology at the front of the migration and favored cell migration toward the wound in 3D matrix (Supplementary Figure S3D).

These results suggested that RhoB silencing in lung cells induces an elongated morphology in a 3D model, correlated with higher cell dynamics and migration. The switch between elongated and rounded cells could be the result of EMT/ mesenchymal-epithelial transition or amoeboid-mesenchymal transition/MAT. In our 3D model, rounded cells did not present obvious amoeboid characteristics such as membrane blebbing or high contractility (Supplementary Figure S1A, Supplementary Movie S1). Thus, to assess whether the elongated shape and invasiveness after RhoB modulation were the result of an EMT process, we further evaluated the expression of several EMT markers in BEAS-2B cells following RhoB knockdown. RhoB silencing significantly increased the mRNA levels of Slug, but surprisingly decreased the mRNA expression of Snail (Figure 2a). Similar results were observed at the protein level (Figure 2b). Consistent with the stimulation of Slug expression, E-cadherin mRNA and protein expression levels were significantly downregulated (Figures 2a and b). Moreover, we observed a strong induction of Slug and inhibition of $\mathrm{E}$-cadherin expression in

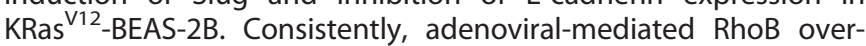
expression led to the opposite (Supplementary Figure S4A).

Interestingly, the elongated lung cells H1975, Calu-1 and H1650 exhibited Snail and/or Slug expression, whereas no expression was detected in the rounded cells H520, H460, A549 and H4006 (Supplementary Figure S4B), bearing out that elongated cells have undergone EMT.

We then examined whether siRhoB-mediated Slug induction accounted for the morphological changes and increased invasiveness of cells. The depletion of Slug (Supplementary Figure S4C) totally suppressed the effect of RhoB silencing on the morphological changes and suppressed the invasiveness of cells (Figures $2 c$ and d). This suggested that Slug was required for the effects of RhoB silencing in these cells.

Altogether, these data indicate that RhoB inhibition could promote EMT in lung cells, resulting in increased mesenchymal morphology and invasiveness of BEAS-2B cells through a Slugdependent mechanism.

\section{RhoB depletion induces EMT via activation of an Akt1-Rac1} signaling pathway

Recent publications reported that the Akt pathway controls Slug expression. ${ }^{26-29}$ Moreover, we previously showed that the activation of Akt1 and Rac1 mediates the migration and invasion induced by RhoB silencing in lung cells. ${ }^{22}$ We then assessed the role of Akt1 and Rac1 in the RhoB-dependent EMT control in BEAS-2B using a siRNA co-transfection strategy. As expected, siRNA-mediated RhoB downregulation significantly increased the proportion of mesenchymal cells, whereas Rac1 or Akt1 silencing suppressed the effect of RhoB downregulation on EMT (Figure 3a). Inhibiting Akt and Rac1 activity using Triciribine and NSC23766, respectively, confirmed these results (Figure 3a). This strongly suggested that RhoB loss induced a mesenchymal morphology dependent on Akt1 and Rac1.

To define the signaling axis regulated by RhoB, we first analyzed the effect of Rac1 inhibition on siRhoB-mediated Akt1 activation, either by siRNA knockdown or by pharmacological inhibition. As shown in Figure 3b, Rac1 inhibition failed to prevent Akt1 phosphorylation on $\mathrm{Ser}^{473}$ residue induced by RhoB knockdown, indicating that Rac1 did not participate in Akt1 activation.

We then analyzed the effect of Akt inhibition on Rac1 activation, and showed that Triciribine prevented Rac1 activation triggered 
a
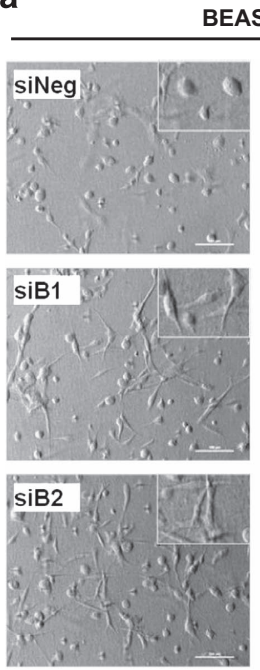

EAS-2B
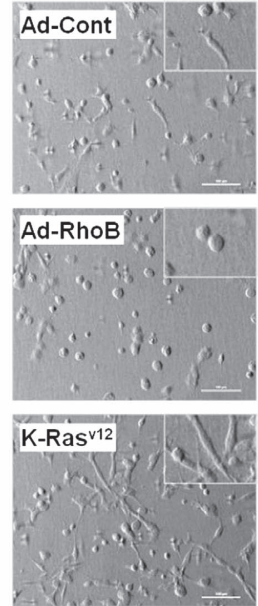

Calu-1
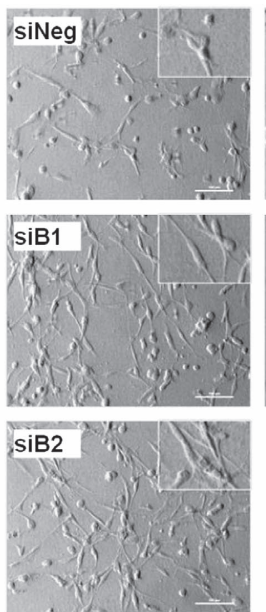
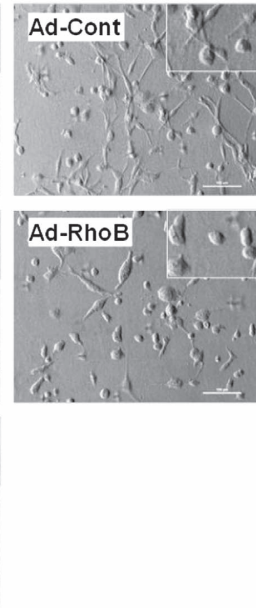

b

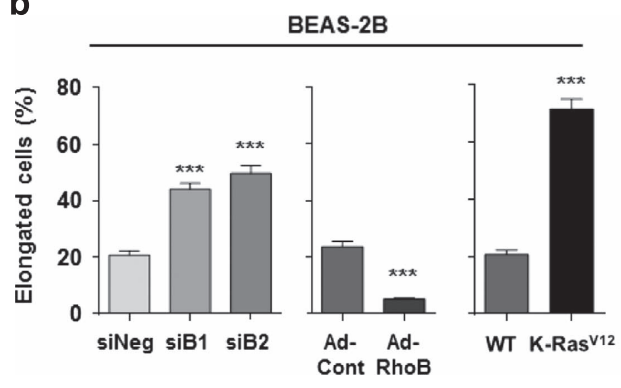

c

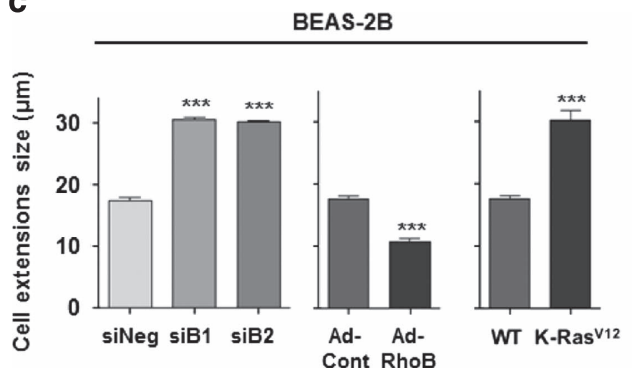

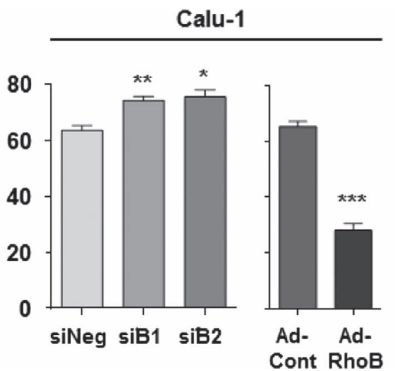

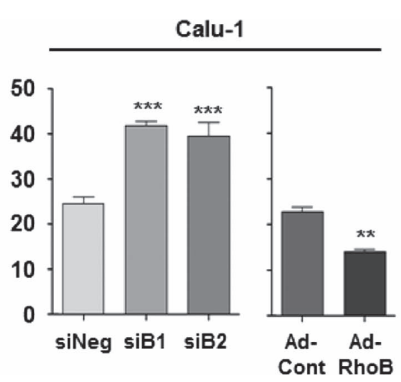

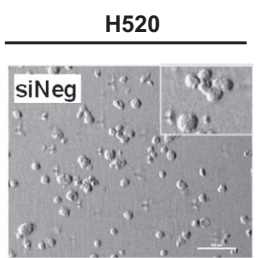
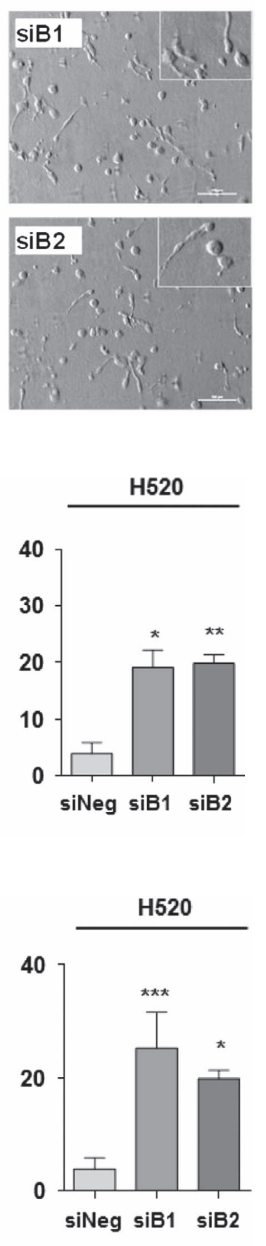

Figure 1. RhoB inhibition induces an elongated morphology of bronchial cell lines in a 3D matrix. (a) As indicated in the panel, different cell lines (BEAS-2B, Calu-1 or H520) were either transformed by the oncogene K-RasV12 (K-Ras V12), transfected with a control siRNA (siNeg), two RhoB siRNAs (siB1 or siB2) or transduced with an adenovirus coding for GFP (Ad-Cont) or RhoB (Ad-RhoB), and were embedded in a 3D matrix. Cells were observed by differential interference contrast (DIC) microscopy. (b) Quantification of the percentage of elongated cells. (c) Measurement of cell-extension size using Nikon NIS-Element software. ${ }^{*} P<0.05 ;{ }^{* *} P<0.01$; ${ }^{* * *} P<0.001$.

by RhoB depletion (Supplementary Figure S5A). To assess whether Akt1 was the only Akt isoform involved in siRhoB-mediated Rac1 activation, we individually inhibited each Akt isoform using specific siRNA, and determined the increased Rac1-GTP/Rac1 ratio resulting from RhoB depletion. Akt1, but neither Akt2 nor Akt3 knockdown, prevented siRhoB-mediated Rac1 activation, suggesting that only the Akt1 isoform was implicated in Rac1 activation (Figure 3c).

NSC23766 is a specific inhibitor of both Rac1-specific guanineexchange factors (GEFs), Trio and Tiam $1 .{ }^{30}$ We therefore determined which of these GEFs was involved in the activation of Rac1 by Akt1. Despite $90 \%$ reduction of Tiam1 mRNA expression (Supplementary Figure S5B), we did not observe any modification of the cell shape driven by RhoB silencing (Figure 3d). By contrast, inhibition of Trio (Supplementary Figure S5B) efficiently reverted siRhoB-induced EMT (Figure 3d). Interestingly, the downregulation of Trio, but not of Tiam1, also dramatically suppressed the invasiveness acquired by RhoB-depleted bronchial cells (Supplementary Figure S5C).

Our results suggested that RhoB depletion induced Rac1 activation through Akt1 and the regulation of GEF Trio, leading to EMT in a 3D context.

RhoB regulates Akt1 activity via the regulation of PP2A phosphatase

We further examined by which mechanisms RhoB controls the Akt phosphorylation level. This kinase might be phosphorylated by phosphoinositide-3-kinase (PI3K), 3-phosphoinositide-dependent protein kinase 1 and mammalian target of rapamycin complex 2, or dephosphorylated by protein phosphatase 2A (PP2A), the phosphatase and tensin homolog (PTEN), or the $\mathrm{PH}$-domain leucine-rich repeat-containing protein phosphatase (PHLPP1/2). 

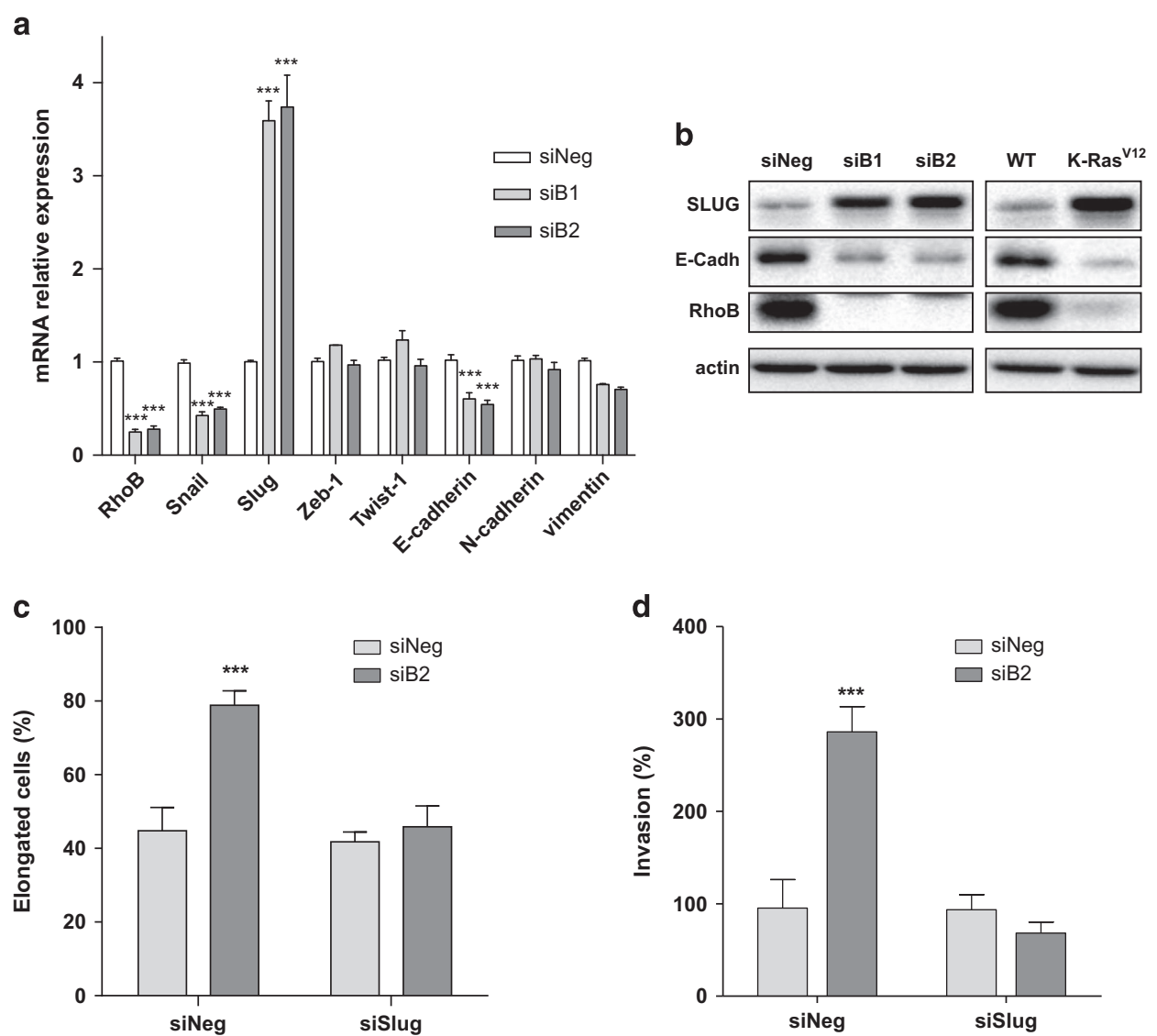

Figure 2. RhoB inhibition increases Slug expression in BEAS-2B cells to trigger invasion. (a) siNeg- and siRhoB-transfected BEAS-2B cells were cultured in 3D for $24 \mathrm{~h}$ and expression of several EMT markers was analyzed by RT-qPCR. (b) Expression of RhoB, Slug and E-Cadherin proteins was assessed by western blot in BEAS-2B cells transfected with siRNA as in A or KRas ${ }^{\mathrm{V} 12}$. BEAS-2B cells were co-transfected with siRhoB (siB2) and siSlug or control siRNA (siNeg), and the percentage of elongated cells (c) and invasiveness (d) was determined as described in the Material and Methods section. ${ }^{*} P<0.05 ;{ }^{* *} P<0.01 ;{ }^{* *} P<0.001$.

Inactivation of PTEN after short hairpin RNA-mediated RhoB inhibition has been previously reported in ovarian OVCAR-3 cells. ${ }^{31}$ However, we did not observe modulation of its activity in BEAS-2B cells (data not shown). Moreover, we have previously observed an inhibitory effect of the PI3K inhibitor LY294002 on bronchial cell migration induced by RhoB knockdown. Thus, we tested the role of RhoB expression on PI3K activity and showed that RhoB silencing by siRNA did not alter PI3K activity (Supplementary Figure S6A).

As RhoB has been shown to interact with the catalytic subunit of PP2A in murine NIH3-3T3 fibroblasts, ${ }^{32}$ we focused on the possible involvement of this phosphatase in the regulation of Akt during the EMT process mediated by RhoB loss, and on the implicated molecular mechanisms. Inhibition of RhoB in bronchial cells using siRNA substantially reduced PP2A activity, as evidenced by the phosphatase-activity assay on immunoprecipitated PP2Aca, whereas RhoB overexpression induced a significant increase in PP2A activity (Figure $4 a$ ). RhoB silencing also increased phosphorylation of the PP2A catalytic alpha subunit (PP2Aca) on tyrosine 370 , which is known to inhibit phosphatase activity of the complex $^{33}$ to a similar degree to that observed with serum treatment (Supplementary Figure S6B).

To determine the implication of PP2A in the EMT process driven by RhoB depletion, BEAS-2B cells were transfected with two different PP2Aca siRNAs, which efficiently decreased both mRNA levels and phosphatase activity (Supplementary Figures S6C and D), and we assessed the impact of PP2A loss on 3D morphological cell changes caused by RhoB overexpression. As previously observed, RhoB overexpression induced a significant decrease of the mesenchymal proportion in control cells, but had no effect in PP2A-depleted cells (Figure 4b), strongly supporting the hypothesis that $\mathrm{RhoB}$ induced a $3 \mathrm{D}$ morphological reorganization through PP2A regulation.

To further investigate the molecular mechanisms by which RhoB regulated PP2A activity in bronchial cells, we first aimed to determine whether these two proteins could interact in human lung epithelial cells, as previously reported by Lee et al. ${ }^{32}$ in the murine NIH3T3 fibroblast cell line. Using a glutathione S-transferase (GST) pull-down assay, we showed that RhoB, but not the closely related GTPase RhoA, efficiently bound PP2A catalytic subunit a (PP2Aca) present in the BEAS-2B cell lysate (Figure $4 \mathrm{C}$ ), and also bound the isolated recombinant human protein (Figure $4 \mathrm{~d}$ ), thus indicating a direct interaction between both proteins. This interaction was confirmed by $\mathrm{CO}^{-}$ immunoprecipitation of endogenous proteins using either a RhoB or a PP2Aca antibody in BEAS-2B cell lysates (Figure 4e). These results suggest that $R$ hoB regulated $P P 2 A$ activity by direct interaction with its catalytic subunit in BEAS-2B cells.

RhoB regulates PP2A activity by regulating the binding of the B55 regulatory subunit

PP2A holoenzymes are composed of catalytic (C), scaffold (A) and regulatory (B) subunits. Specificity and activity of the heterotrimer are defined by the binding of the $B$ regulatory subunit. To date, at least 25 different B subunits have been identified in mammalians and can be classified into four different 
a

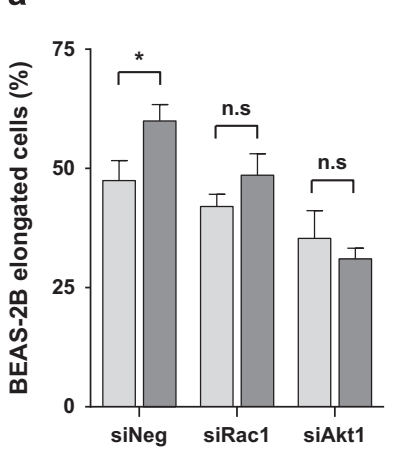

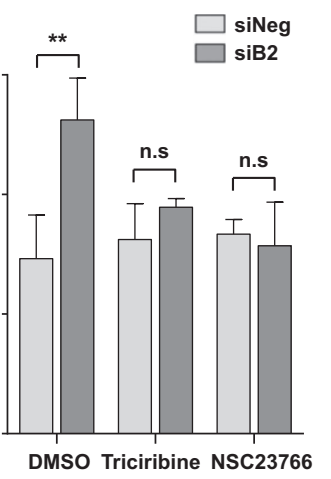

DMSO Triciribine NSC23766 b

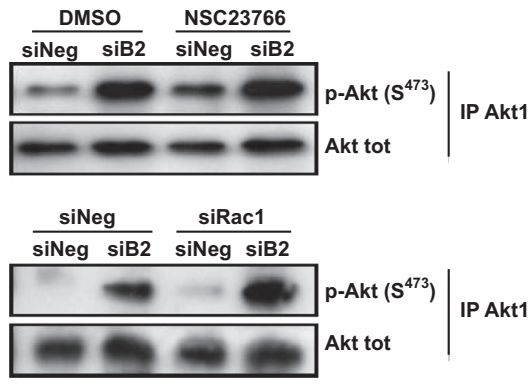

C

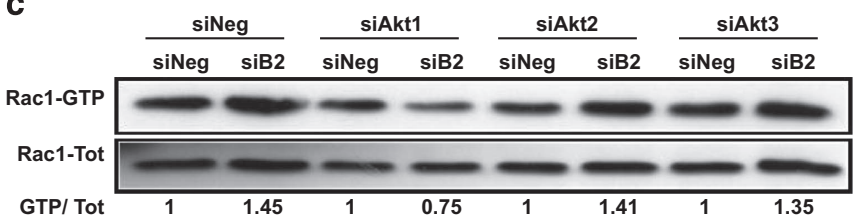

d

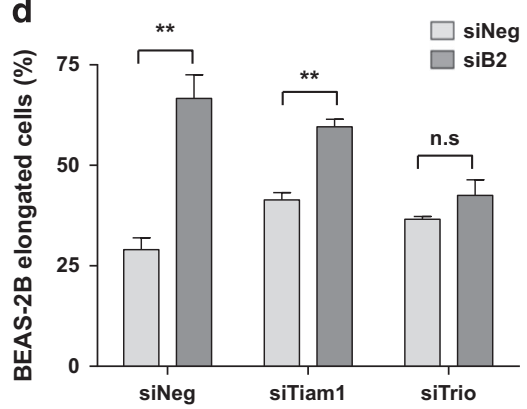

Figure 3. RhoB inhibition induces Rac1 activation via Akt1 regulation. (a) Percentage of elongated BEAS-2B cells embedded in a 3D matrix after being transfected with either a control siRNA (siNeg) or a RhoB siRNA (siB2), and co-transfected with a control siRNA (siNeg), a Rac1 siRNA (siRac1), a Akt1 siRNA (siAkt1) or pretreated or not with the Rac1 inhibitor NSC23766 or the Akt inhibitor Triciribine was determined. (b) BEAS-2B cells were treated with NSC23766 or transfected with a Rac1 siRNA, lysed and Akt1 was immunoprecipitated to assess its phosphorylation state on ser473 by immunoblotting. (c) BEAS-2B cells were transfected with either a control siRNA (siNeg) or a RhoB siRNA (siB2) and were co-transfected with three siRNAs that specifically targeted the different Akt isoforms (siAkt1, 2 and 3). Rac1-GTP was isolated by GST pull down and was revealed by immunoblotting. (d) Percentage of elongated cells embedded in a 3D matrix after being transfected with either a control siRNA (siNeg) or a RhoB siRNA (siB2) and co-transfected with a control siRNA (siNeg), a Tiam 1 siRNA (siTiam1) or a Trio siRNA (siTrio). ${ }^{*} P<0.05 ;{ }^{* *} P<0.01 ;{ }^{* * *} P<0.001 ;$ n.s, non significative.

families-the B (commonly known as B55/PR55), the $\mathrm{B}^{\prime}$ (B56/ PR61), the $B^{\prime \prime}$ (PR48/PR72/PR130) and the $B^{\prime \prime \prime}$ (PR93/PR110). ${ }^{34}$ Although multiplicity of possible combinations offers a great number of putative active complexes, association of PP2A with B55 or B56 subunits appears to confer specificity for Akt targeting. ${ }^{35-37}$

Both subunits were expressed in BEAS-2B cells as revealed by western blot analysis (Supplementary Figure S7A). However, only B55 was able to bind PP2ACa in co-immunoprecipitation assays (Supplementary Figure S7A), suggesting a preferential role of the B55 subunit in BEAS-2B cells. Moreover, siRNA-mediated B55 inhibition led to a significant increase in Akt phosphorylation on both Thr308 and Ser473 (Figure 5a).

Next, to assess whether RhoB was involved in the regulation of Akt1 by B55-associated PP2A, a GST pull-down assay was first performed in a BEAS-2B cell lysate using RhoB as bait. RhoB did not bind B56 (Supplementary Figure S7B) but interacted with B55, as well as with Akt1, indicating the existence of a complex formed by RhoB, PP2ACa, B55 and Akt1 (Figure 5b). Moreover, RhoB depletion resulted in a significant decrease in the PP2ACa-B55 interaction, whereas RhoB overexpression increased the proportion of B55 bound to PP2A (Figure 5c). Furthermore, the RhoB-induced increase of PP2A activity was fully prevented in B55- but not in B56-depleted cells (Figure $5 \mathrm{~d}$ and Supplementary Figure S7C).

Finally, the role of the B55 subunit in the RhoB-dependent 3D morphological changes and invasion was evaluated. B55, but not B56 inhibition by siRNA, significantly suppressed the mesenchymal-epithelial transition and completely reversed the decreased invasion induced by RhoB overexpression (Figures $5 e$ and $f$, Supplementary Figures S7D and E). Altogether, these results support the hypothesis of a nonparticipation of the B56 subunit in the RhoB-mediated regulation of PP2A, and show that RhoB regulates bronchial cell $3 D$ morphology by modulating $P P 2 A$ activity in a specific B55-dependent mechanism.

\section{DISCUSSION}

In the present study, we provide evidence that RhoB depletion, which occurs during lung cancer progression, ${ }^{18}$ favors mesenchymal migration in a 3D matrix, via the regulation of phosphatase PP2A activity, by limiting the binding of its regulatory subunit B55. This leads to activation of the Akt1-Trio-Rac1 signaling axis.

Herein we used a 3D culture model to more accurately match the structure of native tissue, and thus to better characterize the migration mode of tumor cells and underlying mechanisms. We have shown that in these conditions lung tumor cells could adopt distinct morphologies, which could reflect different migration modes. Some cell lines displayed a fibroblast-like morphology with long protrusions, whereas some others adopted a rounded shape. RhoB expression was significantly lower in elongated cells compared with rounded cells, suggesting a role for this GTPase in the control of cell morphology in 3D. This hypothesis was strengthened as we observed that RhoB loss by either siRNA or KRas ${ }^{V 12}$ transfection induced a transition toward a more elongated shape with lengthening of cell protrusions along with a higher cell dynamic and increased invasiveness, as we have previously reported. ${ }^{22}$ Consistently, RhoB overexpression induced a more rounded phenotype and decreased invasiveness.

These data prompted us to investigate whether this morphological change, associated with the acquisition of invasive properties, could be the result of an EMT. Indeed we confirmed 

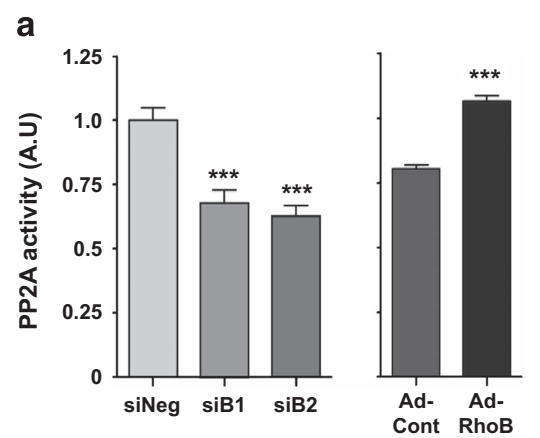

C

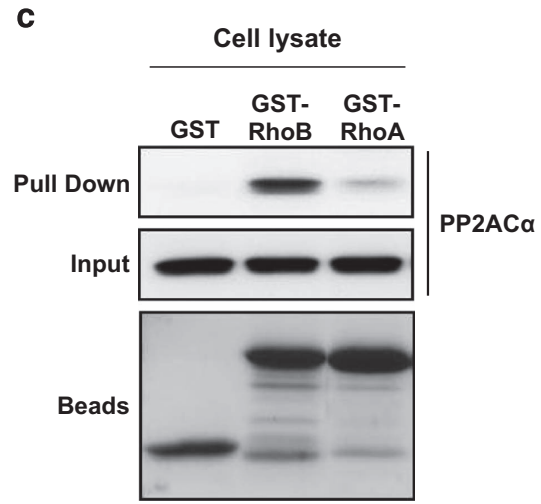

e

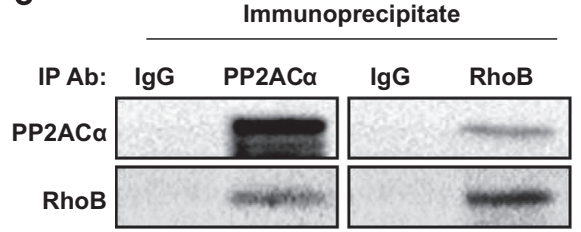

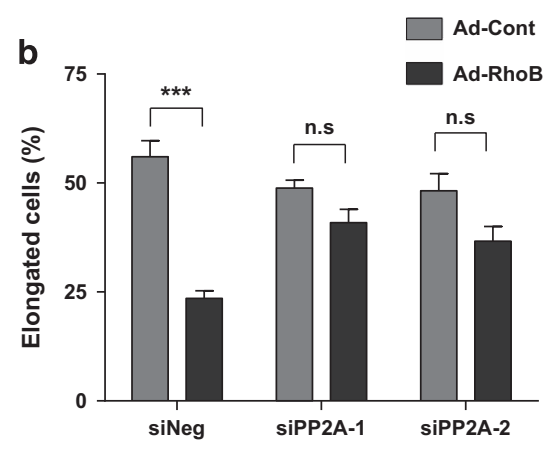

d
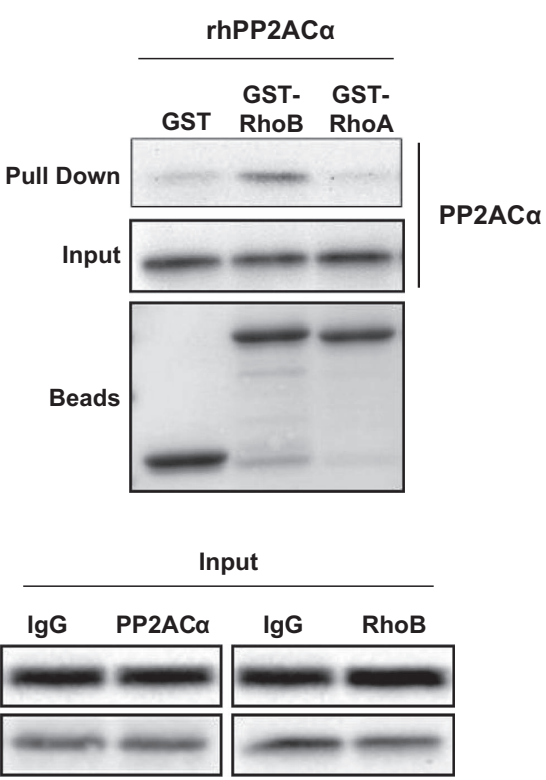

Figure 4. RhoB inhibits the mesenchymal phenotype via PP2A interaction and regulation. (a) PP2A activity was determined in BEAS-2B cells after transfection with either a control siRNA (siNeg) or a RhoB siRNA (siB1 or siB2), or transduced with an adenovirus coding for the GFP (AdCont) or for RhoB (Ad-RhoB). (b) Percentage of elongated BEAS-2B cells embedded in a 3D matrix after being transduced by an adenovirus coding for the GFP (Ad-Cont) or for RhoB (Ad-RhoB) and transfected with a control siRNA (siNeg) or two different PP2A siRNAs (PP2A-1 and PP2A-2). (c) A GST pull-down assay was performed by incubating GST, GST-RhoB or GST-RhoA beads with $500 \mu \mathrm{g}$ of BEAS-2B cell extracts or (d) with $500 \mathrm{ng}$ of human recombinant PP2Ac $\alpha$, and the PP2Ac $\alpha$-bound fraction was assessed by western blot analysis. (e) RhoB-PP2Aco interaction was analyzed by co-immunoprecipitation experiments within the BEAS-2B cell lysates, with either an antibody against RhoB or against the catalytic subunit alpha of PP2A, as revealed by western blotting. ${ }^{*} P<0.05 ;{ }^{* *} P<0.01 ;{ }^{* * * P}<0.001$.

that EMT triggered the morphological changes observed in RhoBdepleted cells, as expression of the EMT-TF Slug was increased and the epithelial marker E-cadherin was inhibited after RhoB depletion. We also observed that Slug and Snail were only expressed in elongated cells, whereas no or weak expression was detected in rounded cells.

Altogether, our data suggest that RhoB loss observed during lung cancer progression ${ }^{18}$ could induce EMT of tumor cells and drive metastatic dissemination. The loss of RhoB expression could be an adaptive response favoring the aggressiveness and development of metastases in lung cancer. We previously reported that RhoB knockdown significantly increased the dissemination of lung metastasis in a murine model. ${ }^{22}$ More recently, we have shown that $\mathrm{RhoB}$ is not only a strong prognostic factor in NSCLC but is also critical for the acquisition of an aggressive phenotype of adenocarcinoma. ${ }^{38}$ However, in vivo experiments have to be performed to determine the relationship between the cell morphology and metastatic dissemination to clarify the role of RhoB in these complex processes. Whether the $3 \mathrm{D}$ cell shape is correlated with invasiveness is still not clear. Indeed, cancer cell invasion appears to be dependent on the cell type and to be a heterogeneous and adaptive process regarding notably the surrounding microenvironment, as well described by Friedl et al. $^{3}$ It could not be excluded that RhoB acts as a metastasis suppressor independently of the morphological changes. Notably, it was reported that RhoB has a critical role in angiogenesis and controls stromal endothelial cells during breast tumorigenesis. 23,39

Among the transcriptional factors involved in mesenchymal migration, we observed that Slug, one of the most thoroughly investigated EMT regulators in lung cancer, ${ }^{40}$ was strongly induced following RhoB loss. In parallel, E-cadherin, epithelial marker of EMT known to be downregulated notably by Slug, showed reduced levels of mRNA and protein. Similarly, it was reported very recently in prostate cancer cells ${ }^{41}$ that RhoB silencing reduced $\mathrm{E}$-cadherin expression only at the protein level, thus by a protein degradation rather than by a transcriptional regulatory mechanism. Interestingly, Slug expression is associated with poor survival in lung carcinoma, ${ }^{42}$ similar to the loss of RhoB expression. ${ }^{43}$ Slug has been shown as a novel invasion-promoting gene notably in lung carcinoma. ${ }^{29,44,45}$ Recent publications reported that Slug expression is regulated through the signaling axis involving Akt. ${ }^{2-29}$ Interestingly, we have demonstrated herein that RhoB depletion induced a mesenchymal phenotype 
a

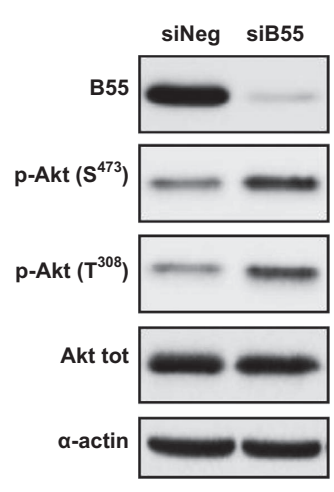

d

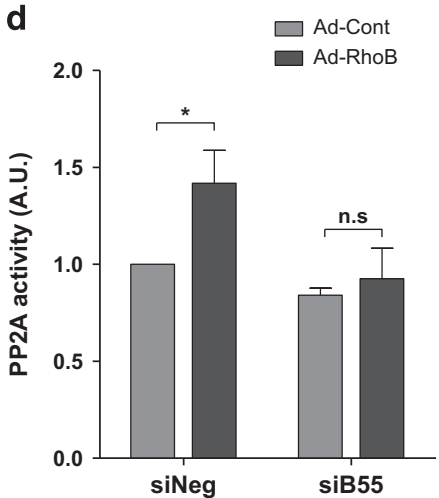

b
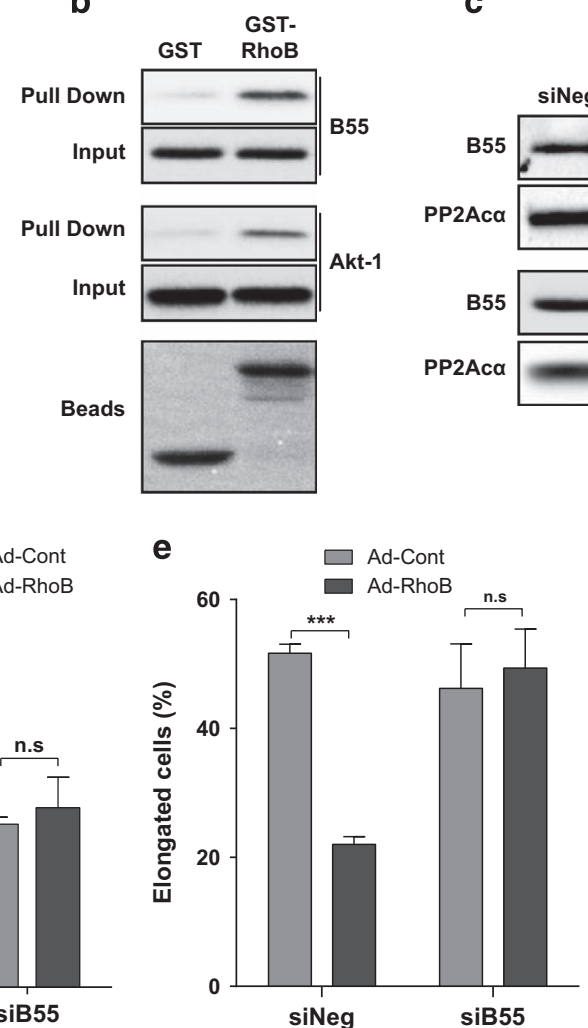

c
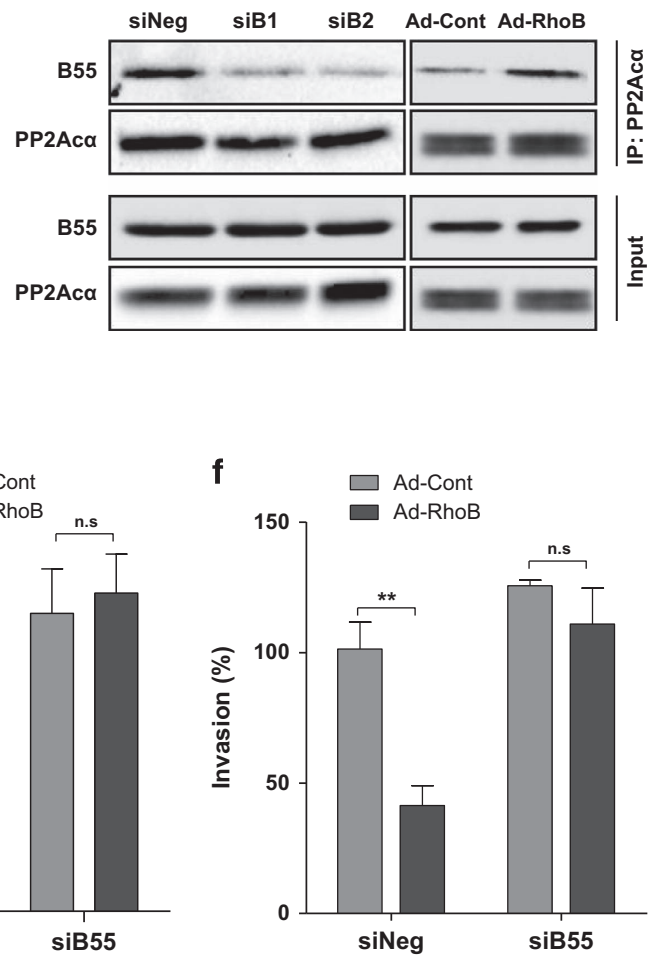

Figure 5. RhoB regulates PP2A activity by modulating its interaction with the B55 regulatory subunit. (a) BEAS-2B cells were transfected with a control siRNA (siNeg) or a siRNA targeted against the B55 regulatory subunit of PP2A (siB55). The phosphorylation state of Akt on S473 and T308 was assessed $48 \mathrm{~h}$ later by western blotting. (b) A GST pull-down assay was performed by incubating GST or GST-RhoB beads with $500 \mu \mathrm{g}$ of BEAS-2B cell extracts, and the B55- or the Akt1-bound fraction was assessed by western blotting using the corresponding antibodies. (c) BEAS-2B cells were transfected with a control siRNA (siNeg) or with two different RhoB siRNAs (siB1 and siB2), or transduced by an adenovirus coding for GFP (Ad-Cont) or for RhoB (Ad-RhoB). As a control, BEAS-2B cells were serum starved for $24 \mathrm{~h}$, and then treated with fetal calf serum for 30 min. Cells were lysed, and the fraction of B55 bound to the PP2A complex was determined by co-immunoprecipitation experiments using an antiPP2Ac $\alpha$ antibody (d) PP2A activity was determined in BEAS-2B cells, which were transduced with an adenovirus coding for the GFP (Ad-Cont) or for RhoB (Ad-RhoB) and then transfected with a control siRNA (siNeg) or a B55 subunit siRNA (siB55). (e and f) BEAS-2B cells were transduced with an adenovirus coding for GFP (Ad-Cont) or for RhoB (Ad-RhoB), and then transfected with a control siRNA (siNeg) or a B55 subunit siRNA (siB55) before being embedded in a 3D matrix (e), or seeded in the upper well of a transwell system (f). Then the percentage of elongated cells $(\mathbf{e})$ and the percentage of invading cells (f) were determined. ${ }^{*} P<0.05 ;{ }^{* *} P<0.01 ;{ }^{* * *} P<0.001 ;$ n.s. non significative.

in a 3D environment via the sequential activation of Akt1 and Rac1 GTPase. In contrast to that observed in leukocytes, ${ }^{46}$ Rac1 did not regulate Akt1 in the bronchial cell model. Our results are in agreement with those of $\mathrm{Xu}$ et al., ${ }^{47}$ who described that the inhibition of the PI3K-Akt pathway in fibroblasts blocked Rac1 activation as well as cell migration.

Altogether, our results propose that the downregulation of RhoB expression in lung cancer $^{18}$ could be critical for the acquisition and maintenance of a Rac1-dependent mesenchymal morphology, probably resulting from an EMT process, and could also be related to the invasive behavior of lung cancer cells.

We have deciphered the mechanism that regulates Rac1 through Akt1 by focusing on its GEFs, Trio and Tiam1. NSC23766 specifically inhibited both of these GEFs as well as the 3D morphological changes induced by RhoB depletion. It is noteworthy that both GEFs have been shown to be involved in the regulation of migration and invasion in several cell types. ${ }^{48-50}$ Trio but not Tiam1 depletion blocked not only the activation of Rac1 but also the EMT induced by RhoB depletion. A recent study shows that Trio mediates TWEAK-induced migration and invasion of glioblastoma cells through Rac1 activation. ${ }^{51}$ The molecular mechanisms of Trio regulation are still unknown. ${ }^{50}$ It was recently reported that Trio tyrosine phosphorylation by the Src kinase Fyn is involved in Rac1 activation and neurite outgrowth in neuroblastoma cells. ${ }^{52}$
In parallel, we examined the molecular mechanisms of Akt regulation by RhoB. The activity of this kinase is finely regulated by either phosphorylation, mainly by 3-phosphoinositide-dependent protein kinase 1 and PDK2, or dephosphorylation, notably by PP2A, PHLPP or PTEN, on two major sites-threonine 308 and serine $473 .{ }^{53}$ We have previously observed an effect of the PI3K inhibitor LY294002 on bronchial cell migration. However, RhoB depletion did not alter PI3K activity. Moreover, whereas inactivation of PTEN after short hairpin RNA-mediated RhoB inhibition was previously reported in ovarian OVCAR-3 cells, ${ }^{31}$ we did not observe modulation of the inhibitory phosphorylation of serine 370 on PTEN in BEAS-2B cells.

Phosphatase PP2A is a tumor suppressor that has been shown to be involved in the regulation of migration in different cancer models, ${ }^{54,55}$ notably in the Lewis lung cancer model. ${ }^{24}$ We evidenced that RhoB regulates PP2A activity, mediating morphological cell changes in a 3D matrix. We have pointed out that RhoB interacts not only with the catalytic subunit alpha of PP2A from bronchial cell lysates, as previously shown in murine NIH3T3 fibroblasts, ${ }^{32}$ but also with purified recombinant protein. This indicates that both proteins bound directly to each other.

The mechanism by which PP2A is functionally inactivated in tumor cells has not been clearly established. The PP2A scaffold and regulatory subunits have been shown to be mutated or aberrantly expressed in many different cancers, 


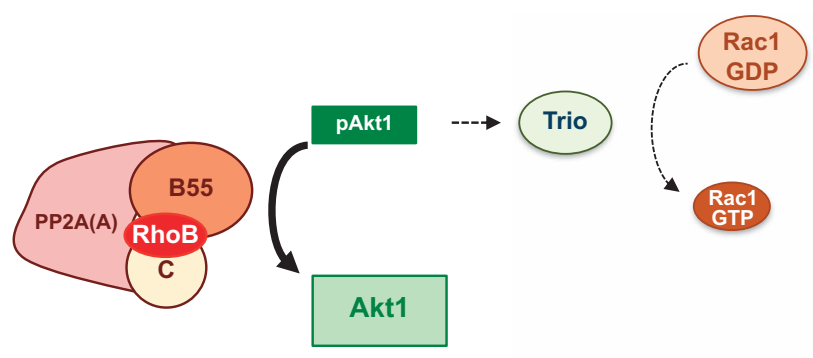

Oncogenic downregulation of RhoB

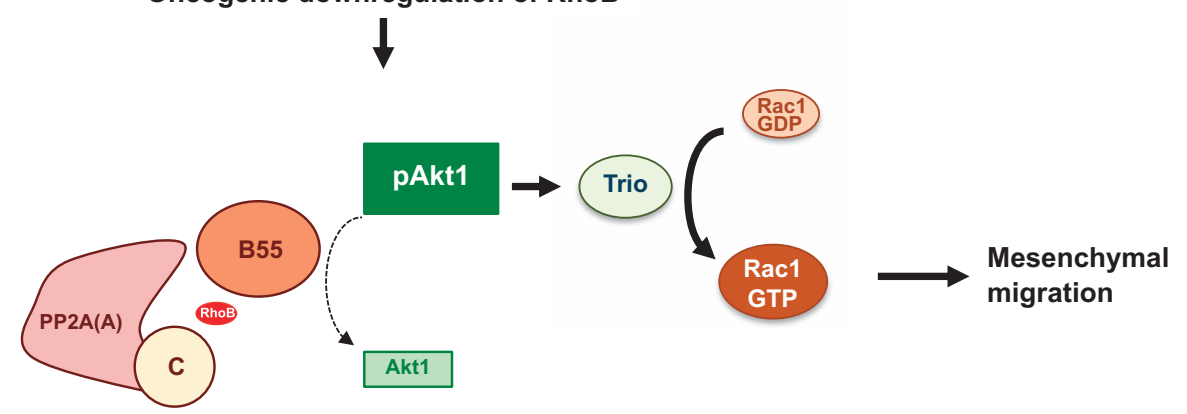

Figure 6. A proposed pathway for the control of cell invasion, migration and 3D morphology by RhoB via regulation of the PP2A-Akt1-Rac1 axis. In bronchial cells, loss of RhoB, a phenomenon frequently observed during lung cancer progression, negatively affects the activity of PP2A, one of the major cellular serine-threonine phosphatases, by promoting the dissociation of its regulatory subunit B55 from the complex. Consequently, the decrease of B55-dependent phosphatase activity of PP2A promotes the activation of its target Akt, which in turn activates Rac1 through its GEF Trio. This increases the capacity of cells to migrate to and invade through the extracellular matrix by regulating the EMT process.

notably in lung cancers. ${ }^{34}$ Herein, we propose RhoB inhibition as a new way to downregulate the tumor suppressor activity of PP2A.

PP2A is a phosphatase formed from three distinct subunits-a catalytic subunit (C), a scaffold subunit $(A)$ and a regulatory subunit (B). This last subunit regulates, spatially and temporally, the activity of the protein, and then allows specificity with different targets of PP2A. ${ }^{34,56}$ Among the 25 regulatory subunits, B55 and B56 have been described to determine the specificity of PP2A for Akt dephosphorylation. ${ }^{35,36}$ In BEAS-2B cells, only B55 could be immunoprecipitated with the PP2A catalytic subunit and its silencing induced Akt phosphorylation. Interestingly, overexpression of miR-222, found in several cancers including lung cancer, ${ }^{57,58}$ has been shown to target and inhibit B55 and increase Akt phosphorylation. ${ }^{59}$ Previous observations of the involvement of B55 in Akt regulation indicate the specific targeting of T308 phosphorylation with no or only a negligible effect on the $\$ 473$ residue of kinase. ${ }^{35}$ In our model, however, B55 inhibition led to increased phosphorylation of both sites, suggesting a full activation of the kinase, and thus strengthening the crucial role of this regulatory subunit in Akt regulation.

Finally, we have provided evidence that RhoB interacts with the B55 subunit together with Akt1 and favors the B55-PP2A interaction. Moreover, B55 silencing reversed the rounded phenotype induced by RhoB overexpression, showing that RhoB could regulate $3 \mathrm{D}$ morphological changes by modulating the recruitment of the regulatory B55 subunit to the PP2A complex in order to reduce the Akt1 phosphorylation level.

Altogether, these results identify RhoB as a new direct regulator of PP2A activity, via the recruitment of the B55 regulatory subunit. During lung cancer progression, the downregulation of RhoB may inhibit PP2A activity following B55 release, leading to the activation of the Akt1-Trio-Rac1 signaling axis, triggering cell plasticity, migration and invasion of bronchial tumor cells (Figure 6).

Our results suggest that the K-Ras oncogenic pathway could be linked to the PP2A tumor suppressor gene pathway through RhoB in lung cancer cell lines. K-Ras-induced downregulation of RhoB could therefore be one of the mechanisms that impair PP2A activity and allow oncogene-transforming functions.

We have demonstrated that RhoB controls PP2A to modulate Akt phosphorylation and $3 \mathrm{D}$ type of migration. A role of RhoB on PP2A activity has also been shown in another cellular process, the regulation of DNA double-strand breaks, ${ }^{60}$ but the involvement of a direct RhoB-PP2A interaction remains to be elucidated. At the time of targeted therapy in lung cancer, the level of RhoB expression should be investigated as a putative surrogate marker of tumor invasion and sensitivity to the PI3K/AKT/mTOR pathway inhibitors.

\section{MATERIALS AND METHODS}

\section{Cell culture and transfection}

Human bronchial epithelial cell lines, BEAS-2B (ATCC, Manassas, VA, USA) CRL-9609, and A549 (ATCC CCRL-185), issued from lung carcinoma, were grown in DMEM supplemented with $10 \%$ fetal calf serum (FCS, Lonza, Ambroise, France). Calu-1 cells (ATCC HTB-54), lung grade-III squamous carcinoma cells, were grown in McCoy's 5a (Gibco, Grand Island, NY, USA) supplemented with $1.5 \mathrm{~mm}$ glutamine and 10\% FCS. H460 (ATCC HTB-177) issued from large cell carcinomas, H520 (ATCC HTB-182) issued from lung squamous carcinomas, and H2935 (ATCC CRL-2869), H23 (ATCC CRL-5800), H1650 (ATCC CRL-5883) and H1975 (ATCC CRL-5908) issued from lung adenocarcinomas were grown in RPMI-1640 (Lonza) supplemented with $10 \%$ FCS. All cell lines were authenticated and tested for mycoplasma contamination recently.

All cell lines were maintained at $37^{\circ} \mathrm{C}$ in a humidified incubator with $5 \%$ $\mathrm{CO}_{2}$. BEAS-2B cells transformed by $\mathrm{K}-\mathrm{Ras}^{\mathrm{V} 12}$ were previously obtained by 
transfection with the pZip-KRas ${ }^{\mathrm{V} 12}$ plasmid. $^{22}$ Transient transfection of small interfering RNA was performed using Oligofectamine (Invitrogen, Grand Island, NY, USA), following the manufacturer's instructions. The different siRNAs are described in Supplementary Table 2. The pharmacological inhibitors Triciribine $(3 \mu \mathrm{m})$ and NSC23766 $(50 \mu \mathrm{m})$ were added $1 \mathrm{~h}$ before and then maintained during the experiment.

\section{Adenoviral transduction}

Cells were plated-up at $24 \mathrm{~h}$ before transduction with an adenoviral vector control that coded for GFP (Ad-Cont) or for RhoB (Ad-RhoB), as described previously, ${ }^{22}$ at a multiplicity of infection of 10 for BEAS-2B cells and 5 for Calu-1 cells.

\section{Cell morphology in a 3D matrix}

Cells were embedded in a 3D matrix constituted of collagen type I $(1.5 \mathrm{mg} / \mathrm{ml}, \mathrm{BD}$ Biosciences) and Matrigel (1/10, BD Biosciences) in EMEM (Eagle's Minimal Essential Medium; $2 \times$, Lonza) at a concentration of $4 \times 10^{4}$ cells $/ \mathrm{ml}$. Drops $(30 \mu \mathrm{l})$ were placed for $1 \mathrm{~h}$ upside down at $37^{\circ} \mathrm{C}$ to allow solidification of the matrix. The complete medium was then added. After $6 \mathrm{~h}$, the cells were fixed with $3.7 \%$ formaldehyde, and cell morphology was observed under a Nikon inverted microscope. Cell extension was measured using Nikon-NIS Element software, and differential interference contrast images were captured using a Nikon DS-Qi2 digital camera.

Invasion

The invasion assay was performed as described previously. ${ }^{22}$

\section{Quantitative real-time reverse transcription PCR}

For mRNA analysis, 3D-cultured cells were recovered by incubating the collagen/matrigel drops with collagenase $(100 \mathrm{U} / \mathrm{ml})$ and dispase $(2.5 \mathrm{U} / \mathrm{ml})$ at $37^{\circ} \mathrm{C}$ for 30 minutes, washed twice with PBS and then lysed with corresponding buffer. Total RNA isolation and processing is described in Supplementary Material, and primer sequences are listed in Supplementary Table 1.

\section{Co-immunoprecipitation and pull-down assays}

Cells were lysed in chilled immunoprecipitation buffer containing $50 \mathrm{~mm}$ Tris- $\mathrm{HCl}$ (pH 7.4), $150 \mathrm{~mm} \mathrm{NaCl}, 1 \% \mathrm{NP}-40,10 \%$ glycerol, $1 \mathrm{~mm}$ EDTA, $1 \mathrm{~mm}$ EGTA and $5 \mathrm{~mm} \mathrm{MgCl}$, supplemented with protease (Sigma, Saint-Louis, MO, USA) and phosphatase (Pierce, Waltham, MA, USA) inhibitor cocktail. The cleared lysate $(500 \mu \mathrm{g})$ was then incubated with $3 \mu \mathrm{g}$ of the indicated antibody and $30 \mu \mathrm{l}$ of protein-A agarose beads (Repligen, Waltham, MA USA) in $1 \mathrm{ml}$ of IP buffer for $3 \mathrm{~h}$ in rotation at $4{ }^{\circ} \mathrm{C}$. After washing three times in cold IP buffer, bead pellets were resuspended in $30 \mu \mathrm{l}$ of $2 \times$ Laemmli buffer and subjected to western blotting. For the pull-down assays, antibody and protein A beads were replaced by $25 \mu$ of GST or the indicated GST-fusion proteins bound to glutathione sepharose $4 B$ beads (GE Healthcare, Waukeska, WI, USA), and the same protocol was followed. For the pull-down assays of purified PP2Aca, the cell lysate was replaced by $500 \mathrm{ng}$ of human recombinant protein (Cayman Chemicals, Tallinn, Estonia) resuspended in IP buffer, and the same protocol was followed.

\section{Quantification of Rac1 activity}

Cells were lysed in lysis buffer ( $50 \mathrm{~mm}$ Tris- $\mathrm{HCl}$ pH 7.4, 1\% Triton-X-100, $500 \mathrm{~mm} \mathrm{NaCl}, 10 \mathrm{~mm} \mathrm{MgCl} 2,2.5 \mathrm{~mm}$ EGTA and $0.5 \%$ sodium deoxycholate) supplemented with protease and phosphatase inhibitors. Cleared lysates were then incubated with GST-PAK for $30 \mathrm{~min}$ at $4{ }^{\circ} \mathrm{C}$. The precipitate was then analyzed by immunoblotting.

\section{Western blot analysis}

For protein analysis, 3D-cultured cells were recovered and lysed as described earlier. Cell extracts were separated on SDS-polyacrylamide gel electrophoresis and electrotransferred onto a PVDF membrane. Blots were probed with antibodies described in Supplementary Material.

PP2A activity

PP2A activity was quantified with a PP2A immunoprecipitation Phosphatase kit (Millipore, Molsheim, France), following the manufacturer's instructions. In brief, the cells were lysed in chilled PP2A lysis buffer (20 mM Imidazole-HCl, $2 \mathrm{~mm}$ EDTA, $2 \mathrm{~mm}$ EGTA) supplemented with protease inhibitors (Sigma). After immunoprecipitation of the PP2A catalytic subunit, the precipitate was incubated with a phosphopeptide (K-R-pT-I-R-R). Then, the free phosphate was quantified by malachite green, and absorbance was read at $620 \mathrm{~nm}$.

Statistical analyses

The mean \pm s.d. values were calculated for each datum point. Differences between groups were analyzed with the Student $t$ test. Data are representative of at least three independent experiments. Data were considered significant at ${ }^{*} P<0.05,{ }^{*} P<0.01$ and ${ }^{* *} P<0.001$.

\section{CONFLICT OF INTEREST}

The authors declare no conflict of interest.

\section{ACKNOWLEDGEMENTS}

We are grateful to Professor Alain Puisieux (CRCL, Lyon, France) for his helpful comments on the manuscript. We thank Rémi Gence for helping with recombinant proteins. This work was supported by grants from INSERM (Institut National de la Santé et de la Recherche Médicale), Groupe de Recherche Institut Claudius Regaud, the Foundation Recherche et Innovation Thérapeutique en Cancérologie and INCa (Institut National du Cancer) France.

\section{REFERENCES}

1 Thiery JP, Sleeman JP. Complex networks orchestrate epithelial-mesenchymal transitions. Nat Rev Mol Cell Biol 2006; 7: 131-142.

2 Puisieux A, Brabletz T, Caramel J. Oncogenic roles of EMT-inducing transcription factors. Nat Cell Biol 2014; 16: 488-494.

3 Friedl P, Alexander S. Cancer invasion and the microenvironment: plasticity and reciprocity. Cell 2011; 147: 992-1009.

4 Ridley AJ. Rho GTPases and cell migration. J Cell Sci 2001; 114: 2713-2722.

5 Karlsson R, Pedersen ED, Wang Z, Brakebusch C. Rho GTPase function in tumorigenesis. Biochim Biophys Acta 2009; 1796: 91-98.

6 Vega FM, Ridley AJ. Rho GTPases in cancer cell biology. FEBS Lett 2008; 582: 2093-2101.

7 Grise F, Sena S, Bidaud-Meynard A, Baud J, Hiriart JB, Makki K et al. Rnd3/RhoE Is down-regulated in hepatocellular carcinoma and controls cellular invasion. Hepatology 2012; 55: 1766-1775.

8 Bustelo XR. Intratumoral stages of metastatic cells: a synthesis of ontogeny, Rho/Rac GTPases, epithelial-mesenchymal transitions, and more. Bioessays 2012; 34: 748-759.

9 Bellovin DI, Simpson KJ, Danilov T, Maynard E, Rimm DL, Oettgen P et al. Reciprocal regulation of RhoA and RhoC characterizes the EMT and identifies RhoC as a prognostic marker of colon carcinoma. Oncogene 2006; 25: 6959-6967.

10 Sanz-Moreno V, Gadea G, Ahn J, Paterson H, Marra P, Pinner S et al. Rac activation and inactivation control plasticity of tumor cell movement. Cell 2008; 135: 510-523.

11 Sahai E, Marshall CJ. Differing modes of tumour cell invasion have distinct requirements for Rho/ROCK signalling and extracellular proteolysis. Nat Cell Biol 2003; 5: 711-719.

12 Chen Z, Sun J, Pradines A, Favre G, Adnane J, Sebti SM. Both farnesylated and geranylgeranylated RhoB inhibit malignant transformation and suppress human tumor growth in nude mice. J Biol Chem 2000; 275: 17974-17978.

13 Liu AX, Rane N, Liu JP, Prendergast GC. RhoB is dispensable for mouse development, but it modifies susceptibility to tumor formation as well as cell adhesion and growth factor signaling in transformed cells. Mol Cell Biol 2001; 21: 6906-6912.

14 Mazieres J, Tillement V, Allal C, Clanet C, Bobin L, Chen Z et al. Geranylgeranylated, but not farnesylated, RhoB suppresses Ras transformation of NIH-3T3 cells. Exp Cell Res 2005; 304: 354-364.

15 Adnane J, Muro-Cacho C, Mathews L, Sebti SM, Munoz-Antonia T. Suppression of rho $B$ expression in invasive carcinoma from head and neck cancer patients. Clin Cancer Res 2002; 8: 2225-2232.

16 Wang S, Yan-Neale Y, Fischer D, Zeremski M, Cai R, Zhu J et al. Histone deacetylase 1 represses the small GTPase RhoB expression in human nonsmall lung carcinoma cell line. Oncogene 2003; 22: 6204-6213.

17 Sato N, Fukui T, Taniguchi T, Yokoyama T, Kondo M, Nagasaka T et al. RhoB is frequently downregulated in non-small-cell lung cancer and resides in the 2 p24 homozygous deletion region of a lung cancer cell line. Int J Cancer 2007; 120: 543-551. 
18 Mazieres J, Antonia T, Daste G, Muro-Cacho C, Berchery D, Tillement V et al. Loss of RhoB expression in human lung cancer progression. Clin Cancer Res 2004; 10: $2742-2750$

19 Wheeler AP, Ridley AJ. RhoB affects macrophage adhesion, integrin expression and migration. Exp Cell Res 2007; 313: 3505-3516.

20 Zhou J, Zhu Y, Zhang G, Liu N, Sun L, Liu M et al. A distinct role of RhoB in gastric cancer suppression. Int J Cancer 2011; 128: 1057-1068.

$21 \mathrm{Ho} \mathrm{HH}$, Chang CS, Ho WC, Liao SY, Lin WL, Wang CJ. Gallic acid inhibits gastric cancer cells metastasis and invasive growth via increased expression of RhoB, downregulation of AKT/small GTPase signals and inhibition of NF-kappaB activity. Toxicol Appl Pharmacol 2013; 266: 76-85.

22 Bousquet E, Mazieres J, Privat M, Rizzati V, Casanova A, Ledoux A et al. Loss of RhoB expression promotes migration and invasion of human bronchial cells via activation of AKT1. Cancer Res 2009; 69: 6092-6099.

23 Kazerounian S, Gerald D, Huang M, Chin YR, Udayakumar D, Zheng N et al. RhoB differentially controls Akt function in tumor cells and stromal endothelial cells during breast tumorigenesis. Cancer Res 2013; 73: 50-61.

24 Jackson J, Meisinger J, Patel S, Lim ZC, Vellody K, Metz R et al. Protein phosphatase-2A associates with the cytoskeleton to maintain cell spreading and reduced motility of nonmetastatic Lewis lung carcinoma cells: the loss of this regulatory control in metastatic cells. Invasion Metastasis 1997; 17: 199-209.

25 Rintoul RC, Sethi T. The role of extracellular matrix in small-cell lung cancer. Lancet Oncol 2001; 2: 437-442.

26 Carpenter RL, Paw I, Dewhirst MW, Lo HW. Akt phosphorylates and activates HSF-1 independent of heat shock, leading to Slug overexpression and epithelialmesenchymal transition (EMT) of HER2-overexpressing breast cancer cells. Oncogene 2014; 34: 546-557.

27 Cordonnier T, Bishop JL, Shiota M, Nip KM, Thaper D, Vahid S et al. Hsp27 regulates EGF/beta-catenin mediated epithelial to mesenchymal transition in prostate cancer. Int J Cancer 2015; 136: E496-E507.

28 Li Y, Jia L, Ren D, Liu C, Gong Y, Wang N et al. Axl mediates tumor invasion and chemosensitivity through $\mathrm{PI} 3 \mathrm{~K} / \mathrm{Akt}$ signaling pathway and is transcriptionally regulated by slug in breast carcinoma. IUBMB Life 2014; 66: 507-518.

29 Lin CH, Wang WC, Kao SH. Der p 2 promotes motility of airway epithelial cell attributing to AKT/GSK3beta-associated epithelial-to-mesenchymal transition. $\mathrm{Mol}$ Cell Biochem 2014; 395: 135-143.

30 Gao Y, Dickerson JB, Guo F, Zheng J, Zheng Y. Rational design and characterization of a Rac GTPase-specific small molecule inhibitor. Proc Natl Acad Sci USA 2004; 101: 7618-7623.

31 Delord JP, Quideau S, Rochaix P, Caselles O, Couderc B, Hennebelle I et al. Trastuzumab induced in vivo tissue remodelling associated in vitro with inhibition of the active forms of AKT and PTEN and RhoB induction in an ovarian carcinoma model. Br J Cancer 2010; 103: 61-72.

32 Lee WJ, Kim DU, Lee MY, Choi KY. Identification of proteins interacting with the catalytic subunit of PP2A by proteomics. Proteomics 2007; 7: 206-214.

33 Chen J, Parsons S, Brautigan DL. Tyrosine phosphorylation of protein phosphatase $2 \mathrm{~A}$ in response to growth stimulation and v-src transformation of fibroblasts. J Biol Chem 1994; 269: 7957-7962.

34 Seshacharyulu P, Pandey P, Datta K, Batra SK. Phosphatase: PP2A structural importance, regulation and its aberrant expression in cancer. Cancer Lett 2013; 335: 9-18.

35 Kuo YC, Huang KY, Yang CH, Yang YS, Lee WY, Chiang CW. Regulation of phosphorylation of Thr-308 of Akt, cell proliferation, and survival by the B55alpha regulatory subunit targeting of the protein phosphatase $2 \mathrm{~A}$ holoenzyme to Akt. J Biol Chem 2008; 283: 1882-1892.

36 Ruvolo PP, Qui YH, Coombes KR, Zhang N, Ruvolo VR, Borthakur G et al. Low expression of PP2A regulatory subunit B55alpha is associated with T308 phosphorylation of AKT and shorter complete remission duration in acute myeloid leukemia patients. Leukemia 2011; 25: 1711-1717.

37 Rocher G, Letourneux C, Lenormand P, Porteu F. Inhibition of B56-containing protein phosphatase $2 \mathrm{As}$ by the early response gene IEX-1 leads to control of Akt activity. J Biol Chem 2007; 282: 5468-5477.

38 Calvayrac O, Pradines A, Rouquette I, Raymond-Letron I, Bousquet E, Lauwers-Cances $\mathrm{V}$ et al. RhoB as a prognostic Bismark in lipidic adenoma. Clin Cancer Res 2014; 20: 6541-6550 In revision.
39 Gerald D, Adini I, Shechter S, Perruzzi C, Varnau J, Hopkins B et al. RhoB controls coordination of adult angiogenesis and lymphangiogenesis following injury by regulating VEZF1-mediated transcription. Nat Commun 2013; 4: 2824.

40 Shih JY, Yang PC. The EMT regulator slug and lung carcinogenesis. Carcinogenesis 2011; 32: 1299-1304.

41 Vega FM, Thomas M, Reymond N, Ridley AJ. The Rho GTPase RhoB regulates cadherin expression and epithelial cell-cell interaction. Cell Commun Signal 2015; 13: 6 .

42 Merikallio H, T T, Paakko P, Makitaro R, Kaarteenaho R, Lehtonen S et al. Slug is associated with poor survival in squamous cell carcinoma of the lung. Int J Clin Exp Pathol 2014; 7: 5846-5854.

43 Calvayrac O, Pradines A, Raymond-Letron I, Rouquette I, Bousquet E, Lauwers Cances $\mathrm{V}$ et al. RhoB determines tumor aggressiveness in a murine EGFRL858Rinduced adenocarcinoma model and is a potential prognostic biomarker for lepidic lung cancer. Clin Cancer Res 2014; 20: 6541-6550.

44 Fenouille N, Tichet M, Dufies M, Pottier A, Mogha A, Soo JK et al. The epithelialmesenchymal transition (EMT) regulatory factor SLUG (SNAI2) is a downstream target of SPARC and AKT in promoting melanoma cell invasion. PLoS One 2012; 7 e40378.

45 Shih JY, Tsai MF, Chang TH, Chang YL, Yuan A, Yu CJ et al. Transcription repressor slug promotes carcinoma invasion and predicts outcome of patients with lung adenocarcinoma. Clin Cancer Res 2005; 11: 8070-8078.

46 Barber MA, Welch HC. PI3K and RAC signalling in leukocyte and cancer cell migration. Bull Cancer 2006; 93: E44-E52.

47 Xu SW, Liu S, Eastwood M, Sonnylal S, Denton CP, Abraham DJ et al. Rac inhibition reverses the phenotype of fibrotic fibroblasts. PLoS One 2009; 4: e7438.

48 van Rijssel J, Hoogenboezem M, Wester L, Hordijk PL, Van Buul JD. The N-terminal $\mathrm{DH}-\mathrm{PH}$ domain of Trio induces cell spreading and migration by regulating lamellipodia dynamics in a Rac1-dependent fashion. PLoS One 2012; 7: e29912.

49 Li Y, Guo Z, Chen H, Dong Z, Pan ZK, Ding H et al. HOXC8-dependent cadherin 11 expression facilitates breast cancer cell migration through Trio and Rac. Genes Cancer 2011; 2: 880-888.

50 Schmidt S, Debant A. Function and regulation of the Rho guanine nucleotide exchange factor Trio. Small GTPases 2014; 5: e29769.

51 Fortin SP, Ennis MJ, Schumacher CA, Zylstra-Diegel CR, Williams BO, Ross JT et al. Cdc42 and the guanine nucleotide exchange factors Ect2 and trio mediate Fn14-induced migration and invasion of glioblastoma cells. Mol Cancer Res 2012; 10: 958-968.

52 DeGeer J, Boudeau J, Schmidt S, Bedford F, Lamarche-Vane N, Debant A. Tyrosine phosphorylation of the Rho guanine nucleotide exchange factor Trio regulates netrin-1/DCC-mediated cortical axon outgrowth. Mol Cell Biol 2013; 33: 739-751.

53 Liao Y, Hung MC. Physiological regulation of Akt activity and stability. Am J Trans/ Res 2010; 2: 19-42.

54 Benefield J, Meisinger J, Petruzzelli GJ, Young MR. Endothelial cell response to human head and neck squamous cell carcinomas involves downregulation of protein phosphatases-1/2A, cytoskeletal depolymerization and increased motility. Invasion Metastasis 1997; 17: 210-220.

55 Nakada N, Kuroda K, Kawahara E. Protein phosphatase 2A regulatory subunit Bbeta promotes MAP kinase-mediated migration of A431 cells. Cell Physiol Biochem 2005; 15: 19-28.

56 Sents W, Ivanova E, Lambrecht C, Haesen D, Janssens V. The biogenesis of active protein phosphatase $2 \mathrm{~A}$ holoenzymes: a tightly regulated process creating phosphatase specificity. FEBS J 2013; 280: 644-661.

57 Mao KP, Zhang WN, Liang XM, Ma YR. MicroRNA-222 expression and its prognostic potential in non-small cell lung cancer. ScientificWorldJournal 20142014 908326.

58 Zhang DQ, Zhou CK, Jiang XW, Chen J, Shi BK. Increased expression of miR-222 is associated with poor prognosis in bladder cancer. World J Surg Oncol 2014; 12: 241.

59 Zhang Y, Ma T, Yang S, Xia M, Xu J, An H et al. High-mobility group A1 proteins enhance the expression of the oncogenic miR-222 in lung cancer cells. Mol Cell Biochem 2011; 357: 363-371.

60 Mamouni K, Cristini A, Guirouilh-Barbat J, Monferran S, Lemarie A, Faye JC et al. RhoB Promotes gammaH2AX dephosphorylation and DNA double-strand break repair. Mol Cell Biol 2014; 34: 3144-3155.

Supplementary Information accompanies this paper on the Oncogene website (http://www.nature.com/onc) 